\title{
NEW HORIZONS
}

\section{NEW HORIZONS IN THE TREATMENT OF HIV INFECTION}

\author{
I M Sanne, $F C P(S A), D T M E H$ \\ W D Francois, FCP (SA), DTMEH \\ W F Venter, FCP (SA), DTMEH \\ Clinical HIV Research Unit, University of the Witwatersrand, Johannesburg
}

Clinicians working with HIV-infected patients have witnessed near-miraculous clinical benefits with antiretroviral therapy. In the space of a few years, an almost universally fatal disease has been transformed into a chronic, manageable outpatient condition.

However, the downsides of the drugs were almost immediately apparent. Tolerability, clinical and metabolic side-effects, lack of convenient dosing schedules, viral genetic resistance and cost make the disease very challenging to treat. The recognition of drug toxicities, particularly of the protease inhibitors, has led to a more tempered approach to the previous practice popular in affluent countries of simply putting patients on treatment early on in their disease. The rapid rise of viral resistance in poorly adherent patients has led to the quest for drugs easier to take, and with higher or new resistance barriers.

The prospect of patients being on lifelong and lifesaving medication has been a considerable inducement for pharmaceutical companies to develop safer and better tolerated drugs. A huge amount of research into new drugs, new uses for older drugs, and completely new strategies to assist in mobilising the body's immune defences, is being undertaken.

Most of the recent developments have been with existing classes of drugs, and with entry inhibitors.

\section{NEW COMPOUNDS AND FORMULATIONS OF EXISTING DRUG CLASSES}

\section{PROTEASE INHIBITORS (PIs)}

The search for Pls that are easy to take and have fewer side-effects continues. About to enter the South African market is the lopinavir/ritonavir combination, marketed as Kaletra, which has been tested in clinical trials locally. This drug is administered twice daily, is better tolerated than many of its predecessors, and has a high resistance barrier. It is very useful in salvage regimens, and daily dosing is being investigated. Despite containing ritonavir, which usually requires refrigeration, the formulation can be kept for up to 2 months at room temperature $\left(22^{\circ} \mathrm{C}\right)$. Abbott has already announced that the drug will be available at access pricing to members of the HIV Clinicians Society. Atazanavir, a once-daily PI which has been tested extensively at several sites in South Africa over the last 3 years, also appears to have a good safety profile, with limited triglyceride and cholesterol changes. A major benefit in Africa is that it is heat-stable at high temperatures, a property not shared by other PIs. In vitro evidence of antiviral efficacy of atazanavir to viral isolates resistant to two more Pls supports the current research programme in salvage therapy. The drug is not yet available internationally, but is due for evaluation by the Food and Drug Administration (FDA) soon. Tipranavir is another $\mathrm{Pl}$ in development, and is currently being evaluated in salvage regimens. Development has been hampered by an initial massive pill burden, similar side-effects to other $\mathrm{Pls}$, and the fact that the drug has been handed from one pharmaceutical company to the next. It is the first nonpeptide $\mathrm{Pl}$, which accounts for its unique resistance profile, but licensing is unlikely to occur in the next 2 years. A precursor to amprenavir is currently in phase 3 studies, with the hope of decreasing the pill burden and tablet size.

\section{NON-NUCLEOSIDE REVERSE TRANSCRIPTASE INHIBITORS (NNRTIs)}

The susceptibility of the two commonly available NNRTIs to single resistance mutations remains a major challenge. The drugs are commonly used as first-line therapy because they are potent, easy to take and, despite significant shortterm toxicities, have good long-term tolerance. Currently, the acquisition of the common mutations occurs rapidly if the drug is incorrectly taken. The mutations confer highlevel resistance, and generally mean that the entire class of drugs is not available for future use. Furthermore, the mutation does not result in less 'fit' virus, which can occur with nucleoside analogue mutations.

New drug development in NNRTls includes emivirine, which has now completed phase 3 testing, including in South 
Africa. Possible benefits of emivirine include reduced hepatotoxicity, and absence of skin rash, but commercia viablility of the drug is limited because efficacy appears to be similar to currently registered medication. Investigators in South Africa felt that emivirine did provide good clinical response and treatment efficacy, but its future will depends on the commercial viability.

Second-line NNRTIS, which have resistance profiles different to the two currently available options, are being assessed. DPC 083 is biochemically very similar to efavirenz, but is more potent, with fewer neuropsychiatric side-effects. Very early trials have been encouraging, but the drug has a long development road ahead. Another experimental NNRTI, TMC 125, may also benefit patients with NNRTI resistance mutations, and has shown remarkable efficacy in drug-naïve patients. Again early data are very encouraging, but larger trials are awaited.

A third NNRTI, capravirine, was already in phase 3 development in 2001, when its continued testing on humans was interrupted after animal studies showed vasculitis at dosing levels in excess of the human dose. There are indications from the current patent holder Pfizer that the drug will continue in development, with phase 3 clinical trials planned for 2002. In vitro activity of capravirine includes NNRTI-resistant viruses.

\section{NUCLEOSIDE ANALOGUES}

These drugs continue to form the 'backbone' of the vast majority of antiretroviral combinations.

Ddl (Videx) is an inexpensive and potent nucleoside analogue, commonly used in southern Africa. Monotherapy studies indicate that ddl can reduce viral loads by up to $-2.0 \mathrm{log}$. A new enteric-coated formulation called Videx EC is being investigated, with improved palatability, improved gastrointestinal tolerability, and potentially less peripheral neuropathy. The original ddl, as well as the new formulation, can be used in once-daily dosing. The new enteric-coated formulation still is recommended for dosing on an empty stomach. Overall the benefits of Videx EC and once-daily dosing will lead to increased use of this nucleoside in first-line therapy.

D4T is also being assessed in an extended-release form (Zerit XR), including trials in South Africa. The only real benefit will probably be once-daily dosing, as early data suggest no difference in side-effects. With ongoing concerns about lipoatrophy, mitochondrial toxicity, peripheral neuropathy and lactic acidosis, D4T is being spared internationally in first-line therapy. Clinicians in South Africa cannot afford to ignore this potent nucleoside analogue in view of the access pricing initiatives of Bristol Myers Squibb.
Emtricitabine ('FTC') is a potent, well-tolerated nucleoside analogue derivative of 3TC with similar dosing, efficacy and side-effects. While resistance mutations M184V are associated with resistance to $\mathrm{FC}$, additional potency of FTC in a triple-therapy regimen is associated with $50 \%$ less M184 mutation than a triple-therapy regimen containing 3TC in patients with demonstrated virological failure. It has been extensively used in South African research sites. The FDA is currently reviewing its registration.

GlaxoSmithKline is following its hugely successful Combivir (the combination of AZT and 3TC) with Trizivir, abacavir (Ziagen) added to Combivir, in a single tablet, given twice daily. The drug combination is widely used in the USA, and will be released soon in South Africa. The benefit of a triple nucleoside combination is that two further classes of drugs are available if the drug fails, and the ease of administration is very attractive. Concerns have been raised that abacavir-containing regimens have less efficacy in the face of very high viral loads (>100 000 copies $/ \mathrm{ml}$ ), a common problem in South Africa, where patients often present late and symptomatic. Abacavir's peculiar resistance profile means that the drug should probably be used as the first-line choice, as its efficacy in salvage is poor. The potentially fatal hypersensitivity of abacavir is a side-effect of concern, especially as inexperienced doctors may use the combination drug more readily, as it is easy to prescribe, but miss the hypersensitivity reaction. The cost of this triple therapy combination tablet is limiting its widespread use in resource-poor settings.

Tenofovir was recently approved by the FDA. The drug is a much safer derivative of the ill-fated adefovir, which had significant renal toxicity and was denied approval by the FDA. In the new product the nephrotoxicity has been resolved, with no significant tubular abnormality noted in clinical studies. Experience in South Africa is limited, as its major role has been in drug-experienced patients, although trial results in treatment naïve-patients should be available later this year. The drug is remarkably well tolerated, very potent and easily administered, with resistance to 3TC paradoxically reversing the infrequent tenofovir resistance mutations. Mitochondrial toxicity does not seem to be a problem. It is hoped that it will be licensed for use in South Africa soon. Its place in a future once-daily single combination pill is being explored.

\section{ONCE-DAILY TREATMENT REGIMENS}

Once-daily treatment regimens, with their potential for improved adherence, have been the aim of treatment research. Of the currently available medications only efavirenz is registered for once-daily use. Early studies demonstrate that 3TC can be administered once daily. Both the pharmacokinetic profile and clinical research evidence 
suggest that FIC can be used once daily, but it needs to be passed by the registration committees. Atazanavir, Videx EC and Zerit XR have all been specifically developed for oncedaily treatment, and once-daily dosing of Kaletra is currently being studied.

\section{NOVEL TARGETS}

The life cycle of the virus throws up multiple potential drug targets. The most advanced group of drugs is the so-called entry inhibitors, which are about to be assessed in phase 3 trials.

\section{ENTRY INHIBITORS}

HIV entry into the host cell is mediated initially by the CD4 molecule, and then either the CCR5 or CXCR4 protein. The virus then fuses with membrane using the viral gp-41 envelope protein.

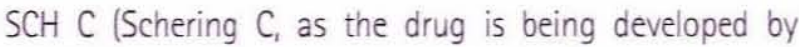
Schering-Plough) is an oral CCR5 antagonist, blocking the second step where the virus binds to the CCR5 receptor. Initial trials showed marked antiviral effects, but possible cardiotoxicity. Dose-ranging studies seem to have addressed this, but more results should be available later this year. Other companies are working on CCR5-blockers, but less is known about the stages of development.

Oral drugs that block CXCR4 are currently being developed, but these are in early development. An intravenous formulation, AMD-3100, has had some antiviral success, but development has been halted because of its toxicity and poor pharmacokinetics.

The 'fusion inhibitors' stop the final binding of the virus to the cell. Several drugs are in development, including T-20, T-1249 and BMS 806. T-20 and T-1249 are only available in injectable form. BMS 806 is available in orally administered form, and recent data are encouraging.

Combinations of the entry inhibitors may be synergistic and are under investigation.

\section{INTEGRASE INHIBITORS}

Integrase is the enzyme that integrates viral DNA into the host cell's DNA, and drug development targeted at the enzyme has been taking place for years. However, prior candidate drugs have been limited by toxicity. Two new compounds are about to enter clinical development, through phase 1 and 2 trials, but clinical use is at best years away.

\section{GENERICS}

With the recent pharmaceutical industry court case and initiatives from the Department of Health, generic manufacture of medications and their importation from other countries have been initiated. Companies that are not bound by patent protection laws are manufacturing combination tablets, using drugs from different drug companies. These are starting to filter into South Africa illegally, as cheap alternatives to available patented products. These products may be granted approval by the South African government in the future. Clinicians should be wary when using these products, as quality assurance is variable with smaller companies. Only limited bioequivalence data and no results of clinical trials are available from generic manufacturers. Cipla, a longestablished Indian company which has manufacturing processes approved by the FDA, the Medicines Control Council (South Africa) and the World Health Organisation, is producing a combination tablet of $\mathrm{d} 4 \mathrm{~T}, 3 \mathrm{TC}$ and nevirapine (Triomune), and other companies will probably follow suit.

\section{STRATEGIES AROUND TREATMENT}

New horizons do not just relate to the new drugs themselves. Treatment strategies for use of the drugs, especially in the resource-constrained environment, continue to develop.

\section{STRUCTURED TREATMENT INTERRUPTIONS}

The realisation that a patient can stop taking antiretrovirals without routine development of resistance has made it possible to develop a number of new treatment strategies to evaluate the efficacy and limit the toxicity of antiviral therapy.

Scientists have postulated that stop-starting therapy in patients with chronic HIV may allow the body's immune system to 'see' the virus in a controlled way, allowing cellmediated and humoral immune responses to HIV to be reacquired. If the body's immune system can be 'autovaccinated' often enough, it may ultimately control the virus without antiretroviral therapy. Initial results have been disappointing in this regard, and the strategy is still being evaluated in trials. However, this strategy may find a place on the basis of savings on drug costs and significantly lower toxicity alone.

There is evidence that structured treatment interruptions (STI) during seroconversion may be beneficial. Current evidence demonstrates that early treatment of HIV followed by treatment interruption during seroconversion leads to improved immune responses to HIV, and is associated with changes in viral and CD4+ set-point after treatment interruption. South African and other guidelines suggest treating these patients, although the specifics of how and for how long is unclear. Unfortunately these patients are difficult to diagnose, 50 treatment during this 
stage is unlikely to be a useful strategy in the vast majority of patients, even if it is found to be effective.

\section{THERAPEUTIC VACCINATION}

This approach is similar to the STI thinking. It is hoped that vaccinating a patient against HIV while they are on HIV treatment may harness more of the immune system in helping to control the virus. The STI experience has tempered enthusiasm for this approach. Early evidence has shown some immunological benefit, but clear clinical benefit is still awaited. A variety of candidate therapeutic vaccines have been tried, without clear evidence of benefit.

\section{THERAPEUTIC DRUG LEVEL MONITORING}

Drug level testing is extensively used in clinical medicine, and includes testing for medicines as widely used as digoxin, the aminoglycosides, lithium, antiepileptics, and cyclosporin. However, the reasons for doing these tests vary - some are used to monitor adherence, others to confirm efficacy, and others to minimise toxicity. Techniques to measure most commercially available PIs and NNRTIs are now well established (the NNRTI's effective levels are intracellular, and therefore cannot be measured).

However, the role of drug testing is still unclear. Assessing adherence may be useful, but the steady state of these drugs is such that simply taking the drug once before an appointment may mean that the patient has a therapeutic level when tested. Toxicity may be related to excessive dosing for some patients, in particular with ritonavirboosted PIs, and may be avoided through therapeutic drug level monitoring. Drug levels of some of the Pls seem to correlate with the side-effect profile, and reducing the dose decreases side-effects without loss of efficacy. Complex drug-drug interactions in patients requiring polypharmacy can be monitored, so that the drug level does not become toxic or subtherapeutic. This information remains limited, but a lot of research is being pursued. One of the difficulties is that most drugs are taken twice daily, making trough and peak interpretations more difficult. The tests are easy for laboratories to do, and will probably be clinically available soon.

\section{GENOTYPE AND PHENOTYPE RESISTANCE TESTING}

These tests are also finding their way into routine clinical practice in the assessment of the 'failing' patient, and as a means of understanding which drugs are less useful, and which can be retained in future regimens. In most cases treatment failure is due to non-adherence with wild-type virus. Genotype testing involves isolation of genetic sequences conferring resistance to drugs. Different mutations confer varying levels of resistance and crossresistance to different drugs. The test is expensive, but relatively quick to do. Expert assessment of the results in conjunction with current and past drug regimens is essential.

Initial cynicism about genotype testing results (and occasional frank disregard) still persists. An expert 'guess' based on knowledge of the patient's past regimens was initially shown to be almost as good a predictor of future success as the test itself. However, the enthusiasts pressed on, and trials now show that interpreting genotyping results in conjunction with expert opinion gives better results than expert opinion alone. Genotype testing sophistication continues to grow as new resistance mutations are discovered and the clinical significance of other mutations become apparent. Expanded access to the tests is limited by the cost and availability of expertise in assessment of results.

Phenotype resistance testing is not yet generally available, but conceptually is more like a routine MIC (inhibitory concentration) for the virus, with growth assessed at different concentrations of the drug. Clinical experience remains limited, it is prohibitively expensive, and the test takes much longer than genotype resistance testing. However, it assesses more than just the most prevalent virus, is easier to interpret, and may give more accurate information about resistance. Its future role is uncertain.

Virtual phenotype' testing uses the results of genotype resistance testing and compares them against a database of phenotype resistance testing on existing similar viruses. It can predict the viral behaviour against different drug combinations. As a sophisticated and constantly evolving interpretation of genotype testing, it has an exciting role.

Current guidelines for resistance testing are limited to treatment-adherent patients demonstrating virological failure. Testing treatment-naïve patients, or patients who are the source of needlestick or other exposure-related injuries, may be indicated before starting antiretrovirals, but is currently controversial.

IDENTIFICATION AND TREATMENT OF SIDE-EFFECTS

The short and long-term side-effects of the antiretrovirals have attracted much unfavourable attention. As all the multi-drug regimen choices are similar in terms of efficacy and dosing schedule, cost and side-effect profile are increasingly defining drug choices.

Lipodystrophy, a complex clinical and metabolic syndrome that has an array of presentations, remains an enigma. The syndrome is seen primarily with the Pls. The increase in visceral fat, abnormal lipid profiles, hypertension and insulin resistance suggests a cardiovascular time-bomb waiting to go off. Interestingly, a massive retrospective 
study recently showed no increase in cardiovascular mortality in patients treated with antiretrovirals, despite the fact that they lived significantly longer. However, the endothelial dysfunction responsible for strokes and myocardial infarction occurs over many years, and we may yet see an epidemic of treatment-related mortality. Treatment of the metabolic parameters is still challenging, as it is not clear whether there is an actual risk. Early indications of metabolic complications in black patients in South Africa indicate that lipodystrophy is associated with diabetes and hypertension rather than lipid changes in this population.

Identifying life-threatening complications before they become serious remains a challenge. Hyperlactataemia has a high mortality if symptomatic, but can be subtle and nonspecific for the unwary clinician. Studies looking at 'safety' parameters to identify patients early are being undertaken. Abacavir hypersensitivity appears in part to be genetically determined. However, routine HLA typing is impractical and expensive, and simpler tests are required to predict this serious complication.

\section{CONCLUSION}

The immediate horizons promise new and betterformulated drugs for South Africans infected with HIV. The role of monitoring of drug levels and resistance patterns is still being determined, and the identification and treatment of side-effects continues to evolve. Huge amounts of research dollars are being poured into the disease, and research appropriate to our region is required.

The prospect of a daily, relatively safe, single combination tablet appears to be on the horizon. In the absence of a cure, making HIV infection an easily managed chronic disease remains a priority. Treatment cost and infrastructure requirements need to be addressed in the resource-poor setting.

\section{BO0K REVIEW}

Handbook of HIV Medicine. By Douglas Wilson, Sudeshni Naidoo, Linda-Gail Bekker, Mark Cotton, Gary Maartens. Pp. 600. Cape Town: Oxford University Press, 2002.

\section{The Oxford Handbook of HIV Medicine for}

Developing Countries is a long-overdue addition to the 'Oxford Handbook' series. It is aimed at all those concerned with the health care of people with HIV, including medical students, nurses, newly qualified doctors, primary care practitioners and hospital doctors, and serves as a practical guide to the assessment and management of HIVrelated problems.

Compiled with the expertise of more than 50 authors, this concise yet comprehensive handbook covers a broad range of topics including diagnostic and management approaches to the common manifestations of HIV in both adults and children, overviews of the virology, immunology and South African epidemiology of HIV, and the holistic care of the HIV-infected person.

Throughout the handbook there is a strong emphasis on the primary care management of the patient with HIV in a resource-limited setting. This is covered in more detail in the chapters on primary care approach, commonly used

drugs in primary care clinics, and home-based care and community liaison.

Highlights include chapters on antiretroviral therapy, oral medicine, nutrition, counselling, the prevention of motherto-child transmission of HIV, infant feeding and palliative

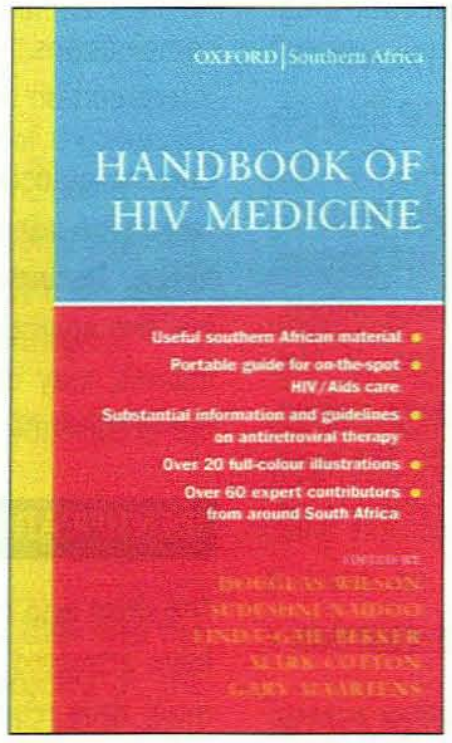
the care of people with HIV in both hospital and community settings in southern Africa and the rest of the developing world.

\section{RUTH CORNICK}

Infectious Disease Clinical Reasearch Unit

UCT Lung Institute

Cape Town issues surrounding HIV care explores many of the dilemmas faced by health care professionals on a daily basis.

Sixty colour photographs and X-rays of common dermatological, oral, respiratory and haematological problems provide a useful reference, and a resource page of national and international HIV-related websites and call-lines ensures access to current developments in HIV therapy, research and sociopolitical issues.

This handbook is endorsed by the Southern African HIV Clinicians Society and is sure to prove an indispensable aid in improving care. A chapter dealing with the ethical 